\title{
Raumbezogene qualitative Sozialforschung
}

\author{
G. Weiss \\ Universität zu Köln, Seminar für Geographie und ihre Didaktik, Gronewaldstraße 2, 50931 Köln, Germany \\ Correspondence to: G. Weiss (g.weiss@uni-koeln.de)
}

Rothfuß, E. und Dörfler, T. (Hrsg.): Raumbezogene qualitative Sozialforschung, Wiesbaden, Springer VS Fachmedien, 310 S., ISBN-13: 978-3-531-16833-3, €30,00, 2013.

In ihren „Konzeptionellen Überlegungen zwischen Geographie und Soziologie" im Prolog zum vorliegenden Sammelband konstatieren die Herausgeber Eberhard Rothfuß und Thomas Dörfler zwei Defizite: methodisch eine Aneignung qualitativer Methoden durch die Geographie ohne raumbezogene Impulse, theoriebezogen ein fehlendes kohärentes Raumkonzept. Gerade im Hinblick auf letzteres streben die Herausgeber mit ihrem Band an, die essenzialistische Raumperspektive und das diskursiv-semantische Verständnis von Raum zusammenzuführen, mithin eine Theorie der Bedeutung des Raumes in der sozialen Welt zu konzipieren. Im Kern rekurrieren die Herausgeber dabei auf die Raumsoziologie von Martina Löw, eine relationale Raumtheorie auf der Grundlage von Simmel, Giddens und Bourdieu. Raum wird demnach durch alltagsweltliche Praxis der Subjekte konstituiert in Form einer Syntheseleistung, in der Elemente der Umwelt (materielle Körper, Images, Szenarien, Atmosphären) als sinnhafte Arrangements zu Räumen zusammengefasst werden. Damit ist aber ein Zugang zu Raum als Teil der sozialen Welt nur über die Subjekte und das Verstehen ihrer mit Sinn versehenen Alltagswelt möglich, was wiederum den Einsatz qualitativer Methoden des interpretativen Paradigmas prädestiniert, um im Subjektiven das Typische zu finden. Gemeinsame, in den Beiträgen des Sammelbandes behandelte Merkmale dieses qualitativen Vorgehens sind entdeckendinduktive Offenheit, Gewinnung von Hypothesen und Theorien aus den erhobenen Daten und Reflexion der Forscherrolle.

Thomas Dörfler liefert zwei Beiträge, einmal zur Theorie von „Milieu und Raum“ sowie zur „Praxis der relationalen Milieuforschung“. Unter Milieus werden alltägliche Lebenswelten der Subjekte mit spezifischen, sozial vorkonstruierten, aber veränderbaren Sinnstrukturen und einer entsprechenden sozialen Semantik verstanden. Zu dieser milieuspe- zifischen Semantik gehört auch eine sozialräumliche Platzierung des eigenen Milieus in einem materiell-imaginären Raum in Abgrenzung zu anderen. Das Ziel relationaler Milieuraumforschung ist es, Raum etablierende Strukturlogiken bzw. Positionierungsleistungen zu Typen (Milieus) zu aggregieren.

Als Beispiel zur Praxis relationaler Milieuraumforschung präsentiert Dörfler seine Erkundung von Milieus im „Feld“ Prenzlauer Berg (Berlin). Aus den Daten teilnehmender Beobachtung und narrativer Interviews wurden über eine Auswertung nach Regeln der objektiven Hermeneutik im Sinne einer Grounded Theory Hypothesen über im Feld vorhandene Milieus aus zunächst maximal kontrastierenden Fällen abgeleitet. Ausgehend von der Grundannahme, dass der soziale Alltag einer Positionierung des Ortsbezugs bedarf, werden Semantiken und Redeweisen, vor allem solche, die zur Abgrenzung von „Anti-Milieus“ dienen, zu einem relationalen Milieuraum verdichtet.

Über „Qualitatives Vergleichen - Zur komparativen Methodologie in Bezug auf räumliche Prozesse" reflektiert Anne Vogelpohl. In der vorwiegend konzeptionellen Betrachtung geht es um methodische Grundüberlegungen des Vergleichens und Spezifika des qualitativen Vergleichens, nämlich die Fokussierung auf ähnliche oder kontrastierende Fallbeispiele mit dem Ziel, Raumkräfte zu erkennen und deren Reichweite einzuschätzen, oder mit anderen Worten den $\mathrm{Zu}$ sammenhang zwischen räumlichen Prozessen und ihrem gesellschaftlichen Kontext zu erkennen. Bei der Datenerhebung selbst sind alle qualitativen Methoden zulässig. Auf Basis der Daten wird eine angemessene Theorie, welche die Analyse weiterer Fälle erleichtern kann, gewählt und gegebenenfalls modifiziert. Abschließend werden die Vorteile qualitativen Vergleichens genannt. Als Beispiel im Hintergrund dient ein Vergleich von zwei Quartieren in Hamburg und New York im Hinblick auf Formen der Flexibilisierung des Alltags in einem urbanen Umfeld.

Peter Dirksmeier liefert im Beitrag ,Zur Methodologie und Performativität qualitativer visueller Methoden" vorwiegend konzeptionelle Überlegungen, ausgehend von einer 
Theorie des Bildes. Er versteht Bilder nicht als Abbild, sondern als Vehikel der Konstitution von Welt. Bilder sind Resultat eines Aushandlungsprozesses zwischen Umweltstimuli und Hintergrundwissen des Subjekts. Dies macht es notwendig, Bilder zu kontextualisieren, d.h. in Entstehungsbedingungen, Motivation und Wahrnehmung des erzeugenden Subjekts einzubetten. Daher müssen bildherstellende qualitative Verfahren mit einer Kontextualisierung der Fotografie über Interviews wie im Verfahren der reflexiven Fotografie kombiniert werden.

Thorsten Fehlberg behandelt in seinem Artikel die „(Re-)Produktion von rechtsextrem dominierten ,Angsträumen“". Dabei handelt es sich vorwiegend um einen konzeptionell fokussierten Ergebnisbericht über Forschungen zu Angsträumen in Ostdeutschland. Im Hinblick auf das alltägliche Erleben von Rechtsextremismus wurden unter Bezug auf das Raumkonzept von Levebvre (mit den Ebenen des wahrgenommenen, konzipierten und gelebten Raums) narrative Interviews mit Experten geführt und diese einer Codierung und Typenbildung unterworfen. Der Autor gibt einige Beispiele für die Produktion von Angsträumen auf den drei Raumebenen durch die beteiligten Gruppen.

Bei Leila Mousa geht es um die ,,(Re-)Produktion und (Re-)Präsentation der Lebenswelt ,Flüchtlingslager““ mit einem Fokus auf methodische Möglichkeiten, sich der für den Forscher relativ fremden Lebenswelt eines palästinensischen Flüchtlingslagers anzunähern. Die Autorin kombinierte dazu Verfahren mit Bezug auf unterschiedliche Alltagsdimensionen, wie teilnehmende Beobachtung, problemzentrierte Leitfaden-Interviews, semiotische Analysen von künstlerischen Produkten nebst Interviews mit deren Produzenten, aktive Teilnahme an Hilfsaktionen sowie die Diskussion von Ergebnissen mit Beforschten in Workshops. Zu jeder Methodik werden kurz ausgewählte Ergebnisse vorgestellt. Zudem reflektiert sie die Problematik von Nähe und Distanz des Forschers zu den Beforschten sowie von Rollenzuweisungen an den Forscher durch die Probanden.

Bei Thomas Uhlendahl geht es um die „Untersuchung von Beteiligungsprozessen in der Raum- und Umweltplanung Ein methodischer Beitrag am Beispiel des Gewässermanagements“. Im Zentrum seines Beitrags steht die Generierung einer Grounded Theory zu Konflikten in Beteiligungsprozessen. Dazu behandelt er zwei Fallbeispiele vertiefend über Aktionsforschung, teilnehmende Beobachtung und problemzentrierte Interviews sowie ergänzend 41 Fälle über standardisierte Befragungen, um über thematische und regionale Vielfalt die Verallgemeinerbarkeit und Plausibilität der Ergebnisse zu verbessern. Aus dem Datenmaterial wurden drei Schlüsselkategorien abgeleitet (Akteursgruppen, Konfliktgegenstände und Interaktionsformen). Von diesen Kategorien ausgehend wurden Bedingungen und Konsequenzen codiert und daraus eine gegenstandsbezogene Theorie mit einem Akteursmodell und einem Modell der Konfliktgegenstände erstellt.
Heidi Kaspar geht es darum, die Wahrnehmung und Nutzung von Parkanlagen auf Basis der Raumsoziologie von Löw verstehend nachzuvollziehen und die Theorie zu konkretisieren. In ihrem Artikel „Raumkonstruktionen aus Erzählungen rekonstruieren. Reflexionen zu einem Forschungsprojekt zur Untersuchung von ,Park-Räumen ““ werden offene Interviews mit Parknutzern über die Praktiken vor Ort als Ausdruck individueller Syntheseleistungen von materiellen Körpern und Atmosphären zu einem Raumtyp „Park“ aufgefasst. Während das Probandenverhalten im Park das Verstehen der individuellen Perspektiven erleichtert, bleibt das Problem sprachlich schwer zu fassender, sinnlich-emotionaler Aspekte der Raumsynthese.

Im Fokus des Beitrags „Eine leibphänomenologische Reflexion über eine nomadische Raumkonzeption" von Eberhard Rothfuß liegt das Verstehen von kulturell fremden Lebenswelten. Dazu wurden die vier Stufen der praktischen Hermeneutik des Fremdverstehens von Sundermeier herangezogen und umgesetzt, um die alltägliche räumliche Ordnung und Orientierung der halbnomadischen, im Norden Namibias lebenden, Himba zu verstehen. Die Erkenntnisse aus distanzierter und teilnehmender Beobachtung sowie aus offenen Interviews wurden aus leibphänomenologischer Perspektive in Anlehnung an Merleau-Ponty nachvollzogen; somit Raum als von Wahrnehmung sowie Bewegungen des Körpers ausgehend konzipiert und an der kosmischen Raumkonzeption der Himba demonstriert.

Eva Kammann berichtet über ihre Forschungen zu „Gender und Raum: Qualitative Zugänge zu Demütigung, sozialer Praxis und ungleichem Alltag in Guatemala" mit Fokus auf der Auswertungsmethodik. Ihr Ziel war es, den Einfluss gesellschaftlicher Machtstrukturen auf den Alltag indigener Frauen nachzuvollziehen. Aus narrativen und problemzentrierten Interviews mit Maya-Frauen wurden mit dem Ziel der Generierung einer Grounded Theory die angesprochenen Phänomene aufgelistet und zu übergeordneten Codes gruppiert. Diesen induktiv gewonnenen Codes wurden wiederum theorienbezogene Konzepte zugeordnet, hier die Theorie des Habitus und der Kapitalsorten von Bourdieu. Abschluss fand die Analyse in der Generierung eines Modells für das Zusammenwirken ursächlicher und intervenierender Bedingungen, Wahrnehmungen und Bewertungen inklusive deren räumlichen Strukturen und Handlungsstrategien.

Ulrike Gerhard und Astrid Seckelmann bieten in ihrem Beitrag mit dem Titel „Kopf oder Zahl? Vermittlung qualitativer Methoden in der humangeographischen Hochschullehre" einen Leitfaden für Lehrende. Darin behandelte Aspekte sind zunächst die Einbettung qualitativer Methodik in eine geographische Fragestellung sowie grundlegende Probleme der Vermittlung qualitativer Verfahren. Am Beispiel der Wahrnehmung des öffentlichen Raums durch Obdachlose wird die praktische Umsetzung qualitativer Verfahren mit Studierenden exemplarisch erläutert mit den Schwerpunkten: Hinführung zum qualitativen Denken, Annäherung an das Feld und Festlegen der Erhebungsmethoden, Formulierung 
und Einüben des Leitfadens, Übung offenen Codierens sowie Diskussion der Ergebnisse mit Dritten bzw. Probanden.

Zum Schluss geht Ulli Vilsmaier („Epilog - Und wo sind wir? Reflexion auf den Ort der/des Forschenden in der raumbezogenen qualitativen Sozialforschung“) auf den Einfluss des Forschers ein. Da der Forschende stets Teil des Raumes ist, den er betrachtet, ist die Genese von Erkenntnissen stets perspektivisch kontextabhängig, einmal in seiner Relation zu den Beforschten, andererseits im Bestreben, die Ergebnisse in eine intersubjektiv nachvollziehbare Form zu bringen. Vilsmaier betrachtet alle Beiträge des Sammelbands auf ihre Reflexion der Position und Einflussnahme des Forschenden. Als Fazit hält er fest, dass es keinen Königsweg für das Verhältnis von Forscher und Gegenstand gebe, denn Nähe sei nötig, um zu verstehen, Distanz, um zu interpretieren und zu kommunizieren. Allerdings bringe eine Positionsanalyse des Forschers größere Genauigkeit und sollte daher fester Bestandteil qualitativer Sozialforschung sein.

Insgesamt gibt der Sammelband einen guten Überblick über die Anwendung konstruktivistischer Raumkonzepte (unter Bezug auf Löw, Giddens, Bourdieu und Levebvre) und verschiedener Formen qualitativer Sozialforschung. Im Hinblick auf die Auswertungsmethodik steht die Grounded Theory von Glaser und Strauss im Vordergrund. Insofern folgen die Beträge keiner einheitlichen Systematik und Konzeptbasis, weder beim Raumverständnis, noch bei der Erhebungs- und Auswertungsmethodik. Da die verwen- deten Konzepte und Theorien nur rudimentäre Erläuterung finden, setzen die Artikel ein hohes Maß an Vorkenntnissen über die etablierten Grundlagen der qualitativen Sozialforschung voraus. Anders ausgedrückt, bieten die Beiträge weder eine grundlegende Einführung in die Raumtheorie noch in die verschiedenen Formen der Erhebungs- und Auswertungsmethodik. Im Hinblick auf letztere ist der Klage von Gerhard und Seckelmann, dass es wenige transparente und kleinschrittige Methodenanwendungen als gute Vorbilder gibt, auch im Hinblick auf den vorliegenden Band zuzustimmen. Die Umsetzung der methodischen Konzepte bleibt an den entscheidenden Stellen häufig zu vage, da allzu oft einer Präsentation ausgewählter Ergebnisse der Vorzug vor einem detaillierten Werkstattbericht gegeben wird. Somit bleiben in einzelnen Beiträgen auch offensichtliche methodische Probleme ausgeklammert, z.B. der Einfluss von Übersetzungen der Interviews oder der Tatbestand, dass selbst bei einer Grounded Theory nur solche Theoriebausteine Verwendung finden, die vorher bekannt sind. Natürlich bleibt die Option, die jeweiligen Originalstudien zu lesen und zu hoffen, dort detaillierte Erläuterungen zu finden. Die Stärke des Sammelbandes liegt vor allem darin, ein Spektrum verschiedener Anwendungsmöglichkeiten und Kombinationen von Methoden und Raumkonzepten zu bieten, mithin Anregungen für eigene Forschungen zu liefern. 